\title{
Grado de desajuste entre las características antropométricas y el mobiliario escolar en una muestra de estudiantes españoles de 6 a 12 años: estudio piloto \\ Degree of mismatch between anthropometric characteristics and school furniture in a sample of Spanish students aged 6-12 years old: A pilot study
}

Prof. Dr. Iván Prieto-Lagea, , Prof. Carlos Ayán ${ }^{a}$, Prof. Dr. Diego Alonso-Fernández ${ }^{a}$,
Mag. Adrián Paramés-González $z^{a, b}$, Mag. Juan Carlos Argibay-González $z^{a, b}$,
Lic. Emma M. Toboada-Solla y Prof. Dr. Alfonso Gutiérrez-Santiagoo,

\section{RESUMEN}

Introducción. El mobiliario escolar influye en la postura sedente que adopta el alumnado en el aula, con efectos en su salud y aprendizaje. Por ello, es preciso determinar la existencia de un posible desajuste entre las dimensiones del mobiliario escolar y la antropometría del alumnado, así como realizar una propuesta de tallas en base a la realidad del aula y las normativas de Galicia y la Unión Europea.

Población y métodos. Un evaluador experto en antropometría realizó las mediciones de peso, talla, altura poplítea, hombro y codo sentado, con un antropómetro, comparándolas posteriormente con las sillas y mesas utilizadas actualmente. Las técnicas de análisis empleadas fueron: descriptivo (medidas de tendencia central), análisis de la varianza de un factor, prueba $t$, prueba de chi cuadrado (en software SPSS $^{\circledR}$ ) y tamaño del efecto ( $d$ de Cohen). El nivel de significación establecido fue de $p \leq 0,05$. Resultados. La muestra se compuso de 108 estudiantes españoles de educación primaria (de entre 6 y 12 años). El 91,7 \% y el 97,2 \% del alumnado emplea, respectivamente, una silla y una mesa que no se ajusta a sus características antropométricas, y utiliza mobiliario con un tamaño superior al que le corresponde. La normativa de tallas de mobiliario que rige actualmente en la población estudiada no es apropiada, al no tener sillas y mesas adecuadas a los primeros cursos de primaria.

Conclusiones. Existe un alto grado de desajuste entre el mobiliario y la antropometría del alumnado. Se propone seguir las tallas de la normativa europea, utilizando varias tallas por curso o mobiliario regulable, para adaptarse a las características antropométricas de todo el alumnado.

Palabrasclave:educación, ergonomía, antropometría, diseño interior y mobiliario, evaluación de necesidades.

http:/ / dx.doi.org/10.5546/ aap.2021.386

Texto completo en inglés:

http: / / dx.doi.org/10.5546/ aap.2021.eng.386
Cómo citar: Prieto-Lage I, Ayán C, Alonso-Fernández D, Paramés-González A, et al. Grado de desajuste entre las características antropométricas y el mobiliario escolar en una muestra de estudiantes españoles de 6 a 12 años: estudio piloto. Arch Argent Pediatr 2021;119(6):386-393.

\section{INTRODUCCIÓN}

Los estudiantes pasan aproximadamente 6,5 horas al día en los centros escolares, ${ }^{1,2}$ y entre un $70 \%$ y un $90 \%$ del tiempo sentados.,4 Esta situación incrementa el riesgo de padecer problemas músculoesqueléticos a una edad temprana, como dolor de cuello, espalda u hombro, como consecuencia del desajuste que suele existir entre las medidas antropométricas del alumnado y las dimensiones del mobiliario escolar. ${ }^{5}$ Por otro lado, el rendimiento académico puede verse igualmente afectado, ya que las posturas corporales incómodas dificultan el aprendizaje, ${ }^{6,7}$ esto causa un aumento de la fatiga que afectará a la capacidad de atención, fundamental en el proceso de aprendizaje. ${ }^{5}$ Teniendo en cuenta que la escuela es un entorno ideal para adquirir hábitos saludables que puedan mantenerse en edad adulta, ${ }^{8}$ se hace necesario emprender acciones para identificar cuáles deben ser las dimensiones ideales del mobiliario escolar.

Esta situación ha sido solventada en algunos países mediante pautas para la estandarización del diseño del 
mobiliario escolar, como por ejemplo en Chile, ${ }^{9}$ Japón ${ }^{10}$ o Corea. ${ }^{11}$ Respecto a España, existen unas directrices relativamente recientes emitidas por la Unión Europea (UE) ${ }^{12}$ que, sin embargo, no parecen imponerse todavía. Esto provoca una falta de actualización en lo referente al diseño y las medidas que debiera presentar el mobiliario escolar. Un ejemplo de esta situación es el caso de la región de Galicia, que cuenta con un catálogo de mobiliario escolar para educación primaria (EP) del año $2007^{13}$ y ninguna talla coincide con el informe de referencia de la UE. ${ }^{12}$

Esta investigación tiene un doble objetivo. En primer lugar, determinar la existencia de un posible desajuste entre las dimensiones del mobiliario escolar y las medidas antropométricas de un grupo de estudiantes de entre 6 y 12 años. En segundo lugar, establecer una propuesta de distribución del mobiliario en base a la realidad del aula y conforme a la normativa de Galicia y de la UE.

\section{POBLACIÓN Y MÉTODOS Población}

Los participantes en este estudio fue alumnado de un centro público de EP situado en una ciudad gallega del noroeste de España, durante el curso académico 2019-20. Se utilizó la técnica de muestreo de conveniencia para facilitar el reclutamiento de estudiantes. Se incluyó en la investigación todo el alumnado de entre 6 y 12 años que presentó el consentimiento informado firmado por los padres o tutores y la autorización de los propios estudiantes.

Para efectuar la investigación, se solicitaron los permisos pertinentes a la dirección del centro. Todas las familias y el alumnado fueron informados sobre los objetivos del estudio, los procedimientos a seguir, la declaración de confidencialidad y los datos de contacto del investigador antes del estudio. En todo momento se respetaron los principios éticos de investigación médica en seres humanos de la Declaración de Helsinki. ${ }^{14}$ El estudio fue aprobado por el comité ético perteneciente a la Facultad de Ciencias de la Educación y el Deporte de la Universidad de Vigo con el código 04/1019.

\section{Evaluaciones}

Para las medidas antropométricas se siguió el procedimiento de otras investigaciones similares. ${ }^{5}$ Los estudiantes fueron evaluados tomando las medidas en el lado derecho (excepto la estatura y el peso), sentados en una silla de altura ajustable con un asiento de superficie horizontal, las piernas flexionadas en un ángulo de $90^{\circ}$ y con los pies totalmente apoyados en un reposapiés ajustable. Durante el proceso de medición, el alumnado estaba descalzo, con pantalón y camiseta corta. Para las mediciones se empleó un antropómetro Cescorf ${ }^{\circledR}$ de $60 \mathrm{~cm}$, homologado por la Sociedad Internacional para el Avance de la Cineantropometría (ISAK, por su sigla en inglés), a excepción de la estatura, que fue medida con un tallímetro Seca ${ }^{\circledR}$ transportable $20-205 \mathrm{~cm}$ y el peso, para el que se utilizó una báscula Tanita UM-076 ${ }^{\circledR}$. Las medidas se realizaron en dos sesiones, fueron registradas en centímetros por un ayudante y fueron realizadas, todas ellas, por un mismo antropometrista. Este hecho permitió minimizar los errores que se producen cuando varios antropometristas realizan las mediciones. ${ }^{15}$ La precisión y la repetibilidad de las mediciones se lograron mediante la capacitación del antropometrista, certificado con nivel ISAK 3 y experiencia previa en este tipo de evaluaciones. Se tomaron un mínimo de dos mediciones de cada parámetro y si los valores hallados variaban más de $0,5 \mathrm{~cm}$ entre ellos, se realizó una medida adicional. Se consideraron las siguientes medidas antropométricas para estimar las dimensiones ideales del mobiliario: ${ }^{16}$

- Estatura: determinada como la distancia vertical entre el piso y la parte superior de la cabeza, medida con el sujeto erguido y mirando hacia el frente en línea recta (plano de Frankfort).

- Altura hombro sentado (HO): distancia vertical desde la superficie en la que se sienta el sujeto hasta el acromion.

- Altura codo sentado (CO): tomada con el codo flexionado en ángulo de $90^{\circ}$. Distancia vertical desde la parte inferior de la punta del codo (olecranon) hasta la superficie sobre la que se sienta el sujeto.

- Altura poplítea (PO): la rodilla deberá estar en flexión de $90^{\circ}$. Distancia vertical desde el suelo hasta la superficie posterior de la rodilla (superficie poplítea).

Los datos antropométricos obtenidos se compararon con las dimensiones del mobiliario para identificar una coincidencia o un desajuste entre ellos. Se definió un desajuste como la incompatibilidad entre las dimensiones del mobiliario actual y la antropometría del alumnado, de acuerdo con las siguientes fórmulas para el cálculo de las medidas ideales: ${ }^{17}$ 
- Altura de la silla (AS): $(\mathrm{PO}+2,5) \cos 30^{\circ} \leq \mathrm{AS} \leq(\mathrm{PO}+2,5) \cos 5^{\circ}$.

- Altura de la mesa (AM): $(\mathrm{AS}+\mathrm{CO} \leq \mathrm{AM} \leq(\mathrm{AS}+\mathrm{CO} \times 0,7396+\mathrm{HO} \times 0,2604)$.

\section{Procedimiento}

Se recogieron las medidas de las sillas y mesas que utilizaba el alumnado en su día a día para el posterior análisis y comparación: ${ }^{17}$

- Altura de la silla actual: distancia vertical desde el suelo hasta el punto medio del borde delantero de la superficie del asiento.

- Altura de la mesa actual: distancia vertical desde el suelo hasta la parte superior del borde frontal del escritorio.

Las mediciones antropométricas se repartieron en dos jornadas consecutivas. Las evaluaciones se realizaron en horario escolar de 9:00 a 14:00 horas, testándose los cursos de primero, segundo y tercero el primer día y los restantes durante el segundo día, durante la primera semana del mes de abril.

\section{Análisis estadístico}

Todos los análisis estadísticos se realizaron utilizando el software Statistical Package for the Social Sciences, versión $20.0^{\circledR}$ (IBM-SPSS Inc., Chicago, USA). Se llevó a cabo un análisis descriptivo, estratificado por curso, de cada una de las variables objeto de estudio a través de medidas de tendencia central (media y desviación típica). La prueba KolmogorovSmirnov confirmó la normalidad de la muestra. Los valores medios de los parámetros obtenidos en los distintos cursos se compararon mediante un análisis de la varianza (ANOVA) de un factor, aplicando una prueba post hoc Tukey-B en el caso de existir diferencias estadísticamente significativas $(p<0,05)$. Dichos valores medios también se compararon entre varones y mujeres, mediante una prueba t para muestras independientes en el caso de variables cuantitativas y una prueba de chi cuadrado en el caso de variables cualitativas.

TABLA 1. Análisis descriptivo y análisis de varianza de un solo factor (ANOVA) de los valores antropométricos del alumnado por curso

\begin{tabular}{|c|c|c|c|c|c|c|}
\hline Curso & $\begin{array}{c}\text { Intervalo } \\
\text { edad (años) }\end{array}$ & $\begin{array}{l}\text { Estatura } \\
(\mathrm{cm})\end{array}$ & $\begin{array}{l}\text { Peso } \\
\text { (kg) }\end{array}$ & $\begin{array}{l}\text { Altura poplítea } \\
\text { (cm) }\end{array}$ & $\begin{array}{l}\text { Altura codo } \\
\text { (cm) }\end{array}$ & $\begin{array}{l}\text { Altura hombro } \\
(\mathrm{cm})\end{array}$ \\
\hline $\begin{array}{l}1^{\circ} \mathrm{EP} \\
\mathrm{n}=11\end{array}$ & $6-7$ & $122,1 \pm 5,5^{\mathrm{a}}$ & $27,2 \pm 3,9^{\mathrm{e}}$ & $29,3 \pm 1,6^{\mathrm{g}}$ & $15,0 \pm 2,0^{\mathrm{i}}$ & $40,1 \pm 2,6^{\mathrm{e}}$ \\
\hline $\begin{array}{l}2^{\circ} \mathrm{EP} \\
\mathrm{n}=13\end{array}$ & $7-8$ & $126,6 \pm 6,0^{a, b}$ & $32,3 \pm 9,2^{e, f}$ & $31,3 \pm 1,1^{b}$ & $15,5 \pm 2,4^{\mathrm{i}}$ & $42,3 \pm 2,9^{e, j}$ \\
\hline $\begin{array}{l}3^{\circ} \mathrm{EP} \\
\mathrm{n}=19\end{array}$ & $8-9$ & $130,3 \pm 6,8^{b}$ & $31,2 \pm 6,1^{\text {ef }}$ & $32,6 \pm 2,2^{b}$ & $14,5 \pm 1,9^{i}$ & $42,1 \pm 2,7^{e, j}$ \\
\hline $\begin{array}{l}4^{\circ} \mathrm{EP} \\
\mathrm{n}=16\end{array}$ & $9-10$ & $138,9 \pm 8,7^{c}$ & $39,7 \pm 13,8^{f}$ & $35,7 \pm 2,6^{c}$ & $15,4 \pm 2,8^{\mathrm{i}}$ & $44,7 \pm 3,8^{j}$ \\
\hline $\begin{array}{l}5^{\circ} \mathrm{EP} \\
\mathrm{n}=21\end{array}$ & $10-11$ & $143,6 \pm 7,5^{c}$ & $39,8 \pm 9,4^{\mathrm{f}}$ & $36,9 \pm 2,0^{\mathrm{ch}}$ & $16,6 \pm 1,6^{h, i}$ & $47,4 \pm 2,7^{\mathrm{k}}$ \\
\hline $\begin{array}{l}6^{\circ} \mathrm{EP} \\
\mathrm{n}=28\end{array}$ & $11-12$ & $150,1 \pm 6,7^{\mathrm{d}}$ & $51,5 \pm 12,3^{\mathrm{d}}$ & $38,5 \pm 1,5^{\mathrm{h}}$ & $18,2 \pm 2,3^{\mathrm{h}}$ & $50,1 \pm 2,7^{\mathrm{d}}$ \\
\hline ANOVA & $\begin{array}{l}\mathrm{F} \\
\mathrm{g} / 1 \\
\text { Sig. }\end{array}$ & $\begin{array}{c}41,66 \\
5 \\
0,00^{*}\end{array}$ & $\begin{array}{c}14,78 \\
5 \\
0,00^{*}\end{array}$ & $\begin{array}{c}60,55 \\
5 \\
0,00^{*}\end{array}$ & $\begin{array}{c}8,34 \\
5 \\
0,00^{\star}\end{array}$ & $\begin{array}{c}31,97 \\
5 \\
0,00^{*}\end{array}$ \\
\hline
\end{tabular}

EP: educación primaria.

${ }^{*} \mathrm{p}<0,05$.

Relación de significatividad entre los cursos académicos:

${ }^{\mathrm{a}} 1^{\circ}$ y $2^{\circ}$ muestran diferencias con los restantes.

${ }^{\mathrm{b}} 2^{\circ}$ y $3^{\circ}$ con los restantes.

${ }^{c} 4$ y $5^{\circ}$ con los restantes.

d $6^{\circ}$ con los restantes.

${ }^{\mathrm{e}} 1^{\mathrm{o}}$ a $3^{\mathrm{o}}$ con los restantes.

${ }^{\mathrm{f}} 2^{\circ}$ a $5^{\circ}$ con los restantes.

${ }^{\mathrm{g}} 1^{\circ}$ con los restantes.

${ }^{\mathrm{h}} 5^{\mathrm{o}}$ y $6^{\mathrm{o}}$ con los restantes.

${ }^{\mathrm{i}} 1^{\circ}$ a $5^{\circ}$ con los restantes.

${ }^{\mathrm{j}} 2^{\circ}$ a $4^{\circ}$ con los restantes.

${ }^{\mathrm{k}} 5^{\circ}$ con los restantes. 
Se realizó una comparación de medias mediante la prueba $t$ para muestras relacionadas para observar las diferencias existentes entre los valores ideales y reales del mobiliario. La comparación de variables cualitativas se realizó mediante la prueba de chi cuadrado. Además, sobre las variables cuantitativas, se analizó el tamaño del efecto mediante la prueba de d de Cohen ( $\mathrm{d}<0,2$ : nulo; $\mathrm{d}=0,2-0,49$ : pequeño; $\mathrm{d}=0,5-0,8$ : moderado; y d >0,8: grande). En todas las pruebas estadísticas se consideró como nivel de significación un valor de $\mathrm{p}<0,05$.

\section{RESULTADOS}

Un total de 110 estudiantes (66 niños y 44 niñas) que estaban matriculados en el centro con edades de entre 6 y 12 años fueron invitados a participar, de los cuales dos no recibieron la autorización familiar. La muestra final estuvo conformada por 108 alumnos (edad media: 9,49 años; $52 \%$ niños y $48 \%$ niñas). Sus características antropométricas, así como la altura del mobiliario escolar registrada se presentan en la Tabla 1.

Los resultados mostraron la existencia de diferencias significativas en los registros antropométricos realizados en función del curso $(p<0,0005)$. No se observaron diferencias significativas por sexo en cada curso.

En la Tabla 2 se muestra la altura media por curso de silla y mesa que empleaba el alumnado antes de la investigación (altura real), así como la

TABLA 2. Comparación entre cursos con análisis de varianza de un factor (ANOVA) de la altura real e ideal de las sillas y las mesas, y comparación por cursos entre la altura real e ideal de las sillas y mesas (prueba $t$ )

\begin{tabular}{|c|c|c|c|c|c|c|c|c|c|c|c|c|c|}
\hline \multirow[t]{2}{*}{ Curso } & \multirow[t]{2}{*}{$\begin{array}{l}\text { Edad } \\
\text { (años) }\end{array}$} & \multirow{2}{*}{$\begin{array}{l}\text { Altura } \\
\text { real silla } \\
\quad(\mathrm{cm})\end{array}$} & \multirow{2}{*}{$\begin{array}{l}\text { Altura ideal } \\
\text { silla }(\mathrm{cm}) \\
\text { Rango } \\
\text { ideal silla } \\
\text { (cm) }\end{array}$} & \multicolumn{2}{|c|}{ Prueba $t$} & \multicolumn{2}{|c|}{$\begin{array}{l}\text { Prueba } d \\
\text { de Cohen }\end{array}$} & \multirow{2}{*}{$\begin{array}{l}\text { Altura } \\
\text { real mesa } \\
\quad(\mathrm{cm})\end{array}$} & \multirow{2}{*}{$\begin{array}{l}\text { Altura ideal } \\
\text { mesa }(\mathrm{cm}) \\
\text { Rango } \\
\text { ideal mesa } \\
(\mathrm{cm})\end{array}$} & \multicolumn{2}{|c|}{ Prueba $t$} & \multicolumn{2}{|c|}{$\begin{array}{l}\text { Prueba } d \\
\text { de Cohen }\end{array}$} \\
\hline & & & & $\mathbf{t}$ & $\mathrm{p}$ & d & $\mathbf{r}$ & & & $\mathbf{t}$ & $\mathrm{p}$ & d & $\mathbf{r}$ \\
\hline $\begin{array}{l}1^{\circ} \mathrm{EP} \\
\mathrm{n}=11\end{array}$ & $6-7$ & $37,1 \pm 1,8$ & $\begin{array}{c}29,6 \pm 1,5^{\mathrm{a}} \\
(27,5 \pm 1,4 / \\
31,7 \pm 1,6)\end{array}$ & 9,041 &, $000^{*}$ & 4,5 & 0,9 & $59,7 \pm 1,5$ & $\begin{array}{c}47,9 \pm 2,5^{\mathrm{e}} \\
(44,6 \pm 2,4- \\
51,1 \pm 2,6)\end{array}$ & 19,127 &, $000^{*}$ & 5,7 & 0,9 \\
\hline $\begin{array}{l}2^{\circ} \mathrm{EP} \\
\mathrm{n}=13\end{array}$ & $7-8$ & $37,8 \pm 2,0$ & $\begin{array}{c}31,4 \pm 1,0^{\mathrm{b}} \\
(29,2 \pm 0,9 / \\
33,7 \pm 1)\end{array}$ & 10,069 &, $000^{*}$ & 4,0 & 0,9 & $60,5 \pm 2,4$ & $\begin{array}{c}50,4 \pm 2,6^{\text {be }} \\
(46,9 \pm 2,5- \\
53,9 \pm 2,7)\end{array}$ & 11,512 &, $000^{*}$ & 4,0 & 0,9 \\
\hline $\begin{array}{l}3^{\circ} \mathrm{EP} \\
\mathrm{n}=19\end{array}$ & $8-9$ & $42,7 \pm 2,7$ & $\begin{array}{r}32,7 \pm 2,0^{b} \\
(30,4 \pm 1,9 / \\
35,0 \pm 2,2)\end{array}$ & 11,227 &, $000^{*}$ & 3,8 & 0,9 & $64,8 \pm 2,9$ & $\begin{array}{c}50,8 \pm 3,0^{\mathrm{b}} \\
(47,2 \pm 2,9- \\
54,4 \pm 3,2)\end{array}$ & 13,292 &, $000^{*}$ & 4,7 & 0,9 \\
\hline $\begin{array}{l}4^{\circ} \mathrm{EP} \\
\mathrm{n}=16\end{array}$ & $9-10$ & $42,4 \pm 1,4$ & $\begin{array}{c}35,6 \pm 2,5^{c} \\
(33,1 \pm 2,3 / \\
38,1 \pm 2,6)\end{array}$ & 11,579 &, $000^{*}$ & 3,4 & 0,9 & $66,0 \pm 0,0$ & $\begin{array}{c}54,8 \pm 4,8^{c} \\
(51,0 \pm 4,6 / \\
58,6 \pm 4,9)\end{array}$ & 9,401 &, $000^{*}$ & 3,3 & 0,9 \\
\hline $\begin{array}{l}5^{\circ} \mathrm{EP} \\
\mathrm{n}=21\end{array}$ & $10-11$ & $42,9 \pm 1,3$ & $\begin{array}{c}36,7 \pm 1,8^{\mathrm{cd}} \\
(34,1 \pm 1,7 / \\
39,3 \pm 1,9)\end{array}$ & 15,214 &, $000^{*}$ & 3,9 & 0,9 & $69,8 \pm 1,8$ & $\begin{array}{r}57,4 \pm 2,7^{c} \\
(53,4 \pm 2,6 / \\
61,4 \pm 2,8)\end{array}$ & 17,195 &, $000^{*}$ & 5,4 & 0,9 \\
\hline $\begin{array}{l}6^{\circ} \mathrm{EP} \\
n=28\end{array}$ & $11-12$ & $42,5 \pm 1,5$ & $\begin{array}{l}38,2 \pm 1,4^{\mathrm{d}} \\
(35,6 \pm 1 / \\
40,9 \pm 1,5)\end{array}$ & 11,430 &, $000^{*}$ & 3,4 & 0,9 & $71,0 \pm 1,4$ & $\begin{array}{c}60,5 \pm 3,0^{\mathrm{f}} \\
(56,4 \pm 2,9 / \\
64,7 \pm 3,0)\end{array}$ & 18,611 &, $000^{*}$ & 4,5 & 0,9 \\
\hline \multicolumn{2}{|c|}{ ANOVA F } & & 60,55 & & & & & 101,03 & 42,49 & & & & \\
\hline & $\mathrm{g} / 1$ & & 5 & & & & & 5 & 5 & & & & \\
\hline & Sig. & & $0,00^{*}$ & & & & & $0,00^{*}$ & $0,00^{*}$ & & & & \\
\hline
\end{tabular}

\footnotetext{
* $\mathrm{p}<0,05$.
}

** Para la comprensión de esta tabla se informa que, a pesar de que la menor talla de silla y mesa del catálogo de primaria de Galicia es 36 y 60 respectivamente, el centro analizado utilizaba mobiliario de educación infantil en los primeros cursos. Por tanto, se observan en la tabla valores medios de la altura real del mobiliario del aula cuyo valor es inferior a las tallas menores del catálogo del mobiliario de esta región española.

Relación de significatividad entre cursos académicos:

a $1^{\circ}$ muestra diferencias con las restantes.

b $2^{\circ}$ y $3^{\circ}$ con las restantes.

c 4 y $5^{\circ}$ con las restantes.

d $5^{\circ}$ y $6^{\circ}$ con las restantes.

e $1^{\circ}$ y $2^{\circ}$ con las restantes.

${ }^{\mathrm{f}} 6^{\circ}$ con las restantes.

Expresión del tamaño del efecto: d y r, $\mathrm{d}<0,2$ : nulo; $\mathrm{d}=0,2-0,49$ : pequeño; $\mathrm{d}=0,5-0,80$ : moderado; y d > 0,8: grande. 
determinada posteriormente como ideal.

En la Figura 1 se muestra el análisis del desajuste del mobiliario escolar por curso. Los resultados muestran la existencia de desajustes entre las características antropométricas del alumnado y la altura del mobiliario empleado. Se encontró que el 91,7\% y el 97,2\% de los participantes en esta investigación utilizaron una silla y una mesa que no se ajustaba a sus dimensiones antropométricas, respectivamente. El alumnado se sienta en una silla entre 2 y $8 \mathrm{~cm}$ más grande de lo que le corresponde y usa una mesa entre 7 y $10 \mathrm{~cm}$ más alta de su talla ideal.

En relación con las medidas ideales de silla y mesa, así como su correspondiente intervalo mínimo y máximo recomendado, se han encontrado diferencias estadísticamente significativas entre los diferentes cursos $(p<0,0005)$. No se aprecian diferencias estadísticamente significativas en cuanto al sexo de los participantes, salvo en segundo curso en la variable altura de la silla ideal $(\mathrm{p}=0,007)$.

FIGURA 1. Grado de ajuste del mobiliario de educación primaria (EP) por curso. A: desajuste de la silla; B: desajuste de la mesa
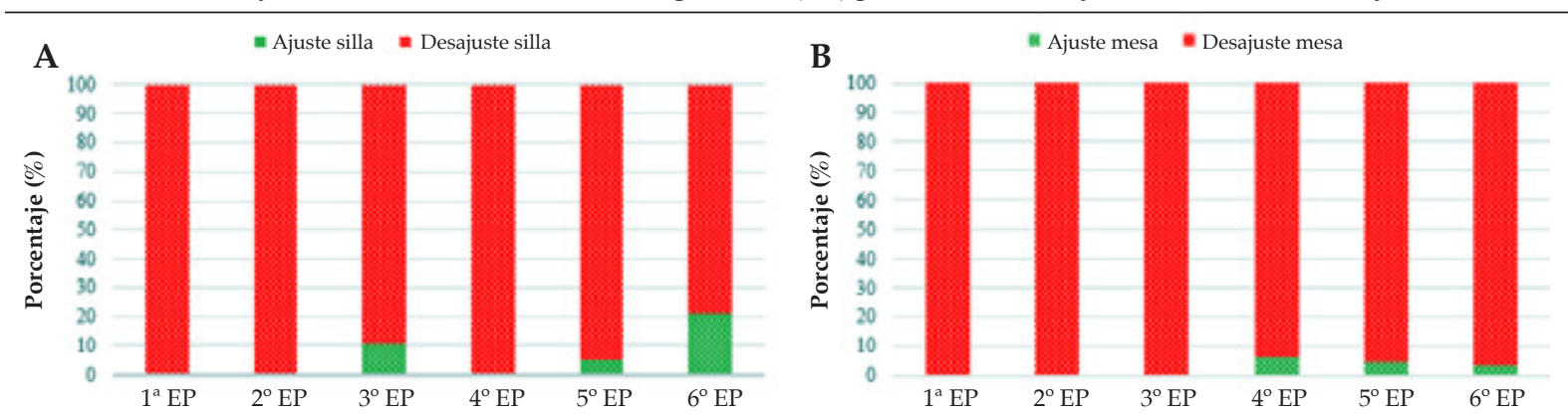

TABla 3. Relación de tallas recomendadas para el centro de análisis mediante la normativa de la región de Galicia y directrices de la Unión Europea

\begin{tabular}{|c|c|c|c|c|c|c|c|c|c|c|c|c|}
\hline \multirow[b]{2}{*}{ Curso } & \multicolumn{3}{|c|}{ Talla silla Galicia } & \multicolumn{3}{|c|}{ Talla silla UE } & \multicolumn{3}{|c|}{ Talla mesa Galicia } & \multicolumn{3}{|c|}{ Talla mesa UE } \\
\hline & Talla & Frec. & Porc. & Talla & Frec. & Porc. & Talla & Frec. & Porc. & Talla & Frec. & Porc. \\
\hline \multirow{3}{*}{ Primero } & & & & & & & M42* & 2 & 18,2 & & & \\
\hline & S28* & 6 & 54,5 & S26 & 3 & 27,3 & M48* & 8 & 72,7 & M46 & 7 & 63,6 \\
\hline & $\mathrm{S} 32^{* *}$ & 5 & 45,5 & S31 & 8 & 72,7 & M54 ${ }^{* *}$ & 1 & 9,1 & M53 & 4 & 36,4 \\
\hline \multirow{2}{*}{ Segundo } & S28* & 1 & 7,7 & & & & M48* & 8 & 61,5 & M46 & 6 & 46,2 \\
\hline & $\mathrm{S} 32^{* *}$ & 12 & 92,3 & S31 & 13 & 100 & M54** & 5 & 38,5 & M53 & 7 & 53,8 \\
\hline \multirow{3}{*}{ Tercero } & S28* & 3 & 15,8 & S31 & 8 & 42,1 & M48* & 11 & 57,9 & M46 & 7 & 36,8 \\
\hline & $\mathrm{S} 32^{* *}$ & 11 & 57,9 & S35 & 10 & 52,6 & M54 ${ }^{* *}$ & 8 & 42,1 & M53 & 12 & 63,2 \\
\hline & S36 & 5 & 26,3 & S38 & 1 & 5,3 & & & & & & \\
\hline \multirow{4}{*}{ Cuarto } & S32** & 3 & 18,7 & S31 & 2 & 12,5 & M48* & 3 & 18,7 & M46 & 3 & 18,8 \\
\hline & S36 & 9 & 56,3 & S35 & 8 & 50 & M54** & 6 & 37,5 & M53 & 6 & 37,5 \\
\hline & S40 & 4 & 25,0 & S38 & 6 & 37,5 & M60 & 7 & 43,8 & M59 & 6 & 37,5 \\
\hline & & & & & & & & & & M64 & 1 & 6,2 \\
\hline \multirow{3}{*}{ Quinto } & S36 & 17 & 81,0 & S35 & 11 & 52,4 & M48* & 1 & 4,8 & M53 & 3 & 14,3 \\
\hline & S40 & 4 & 19,0 & S38 & 10 & 47,6 & M54 & 10 & 47,6 & M59 & 15 & 71,4 \\
\hline & & & & & & & M60 & 10 & 47,6 & M64 & 3 & 14,3 \\
\hline \multirow{3}{*}{ Sexto } & S36 & 10 & 35,7 & S35 & 3 & 10,7 & M54** & 2 & 7,1 & M53 & 1 & 3,6 \\
\hline & S40 & 18 & 64,3 & S38 & 23 & 82,2 & M60 & 20 & 71,5 & M59 & 18 & 64,3 \\
\hline & & & & S43 & 2 & 7,1 & M66 & 6 & 21,4 & M64 & 9 & 32,1 \\
\hline$\overline{\mathrm{F}}$ & & 19.778 & & & 68,667 & & & 38,815 & & & 13,111 & \\
\hline Sig. & & 0.000 & & & 0,000 & & & 0,000 & & & 0,004 & \\
\hline
\end{tabular}

UE: Unión Europea.

* Propuesta de medida que se recomienda emplear pero que no existe actualmente en la normativa del mobiliario de la región de Galicia (ni en educación infantil ni en primaria). Se añaden las tallas de 28 y $32 \mathrm{~cm}$ en la silla y las de 42,48 y $54 \mathrm{~cm}$ en la mesa siguiendo una lógica secuencial de cambio de tamaño que se podría esperar.

** Talla de catálogo de la región de Galicia recomendada para la educación infantil (3-5 años). 
El análisis del tamaño del efecto (prueba $d$ de Cohen) en la altura ideal de la silla indica que las diferencias entre ambos grupos son moderadas $(d=0,74)$. La prueba $t$ para muestras relacionadas entre la altura real del mobiliario y la altura ideal tras el análisis antropométrico, muestra que existen diferencias estadísticamente significativas ( $p<0,0005)$ en todas las comparaciones realizadas. El análisis del tamaño del efecto (prueba $\mathrm{d}$ de Cohen) indica que todas las diferencias encontradas son grandes $(\mathrm{d}>0,8)$.

En la Tabla 3 se presenta la propuesta de mobiliario considerada ideal en base a las características antropométricas del alumnado. Para ello, se tuvo en cuenta el catálogo actual de Galicia ${ }^{13}$ y el documento de referencia de la UE. ${ }^{12}$

En la Figura 2 se ilustra el grado de ajuste evidenciado tras la reorganización del mobiliario escolar en el centro de referencia teniendo en cuenta la normativa de la UE y el catálogo de la región.

\section{DISCUSIÓN}

El presente estudio tuvo como objetivo la evaluación del grado de ajuste del mobiliario en función de la antropometría de un grupo de estudiantes, así como la realización de una propuesta de tallas según la realidad del aula y las normativas de Galicia y la UE.

Las evaluaciones realizadas evidenciaron la existencia de un desajuste entre las características antropométricas del alumnado y la altura del mobiliario escolar; el porcentaje de estudiantes que se sientan en una silla y emplean una mesa, demasiado altas, es excesivamente elevado. Estos desajustes pueden provocar trastornos anatómico-funcionales, problemas en el proceso de aprendizaje y están en la línea de lo reportado en estudios de diferentes continentes. ${ }^{5}$ En Norteamérica observaron que el alumnado utilizaba una silla inadecuada hasta en el $92 \%$ de los casos y una mesa incorrecta en el 95,1 \% de las ocasiones. ${ }^{18}$ Desajustes elevados se han observado también en poblaciones asiáticas, ${ }^{19}$ con un $92,5 \%$ en sillas y $100 \%$ en mesas. En Sudamérica se encontró, en escuelas chilenas, que más del $70 \%$ del alumnado utilizaba una silla incorrecta y en un $100 \%$ de los casos la altura de la mesa era errónea; ${ }^{20}$ también Perú informó de altos niveles de desajuste. ${ }^{21}$ En Europa, trabajos realizados en el norte de Portugal, ${ }^{22}$ informan de niveles de desajuste del $96 \%$ en las sillas y un $76 \%$ en las mesas.

En relación a las normativas en España no existe una regulación estatal, por lo que debe seguir las directrices de la UE; ${ }^{12}$ sin embargo, esta norma no se está siguiendo. Esto puede ser debido a que, por un lado, al estar las competencias educativas transferidas, existe libertad por parte de las regiones del estado para determinar el tipo de mobiliario a utilizar en las escuelas. A su vez, los resultados del estudio muestran que estas administraciones no están siguiendo un criterio antropométrico en las tallas propuestas, y que existe una falta de actualización en lo referente al diseño y medidas que debiera presentar el mobiliario escolar, ya que la norma vigente de Galicia es del 2007. La dificultad de abordar una inversión en nuevo mobiliario por su elevado coste también puede ser uno de los motivos. ${ }^{23,24}$ Los resultados obtenidos indican que para la población objeto de estudio se deberían

FIGURA 2. Nivel de ajuste por curso del mobiliario de educación primaria (EP) por catálogo de referencia de Galicia y de la Unión Europea. A: tallas de las sillas. B: tallas de las mesas

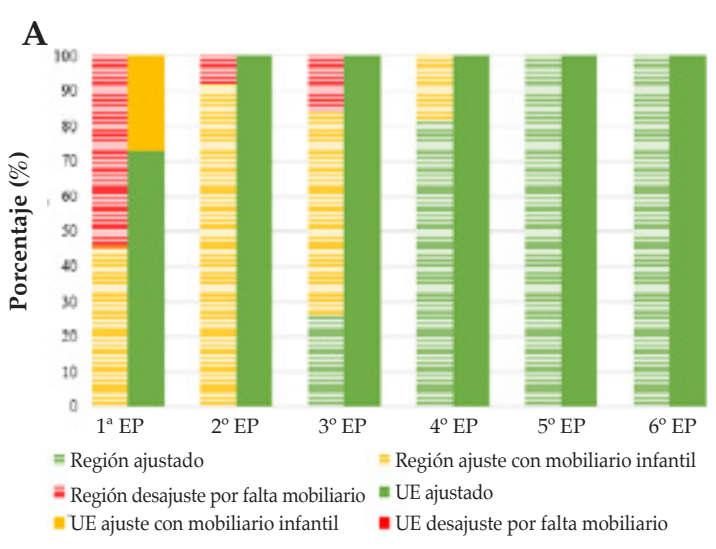

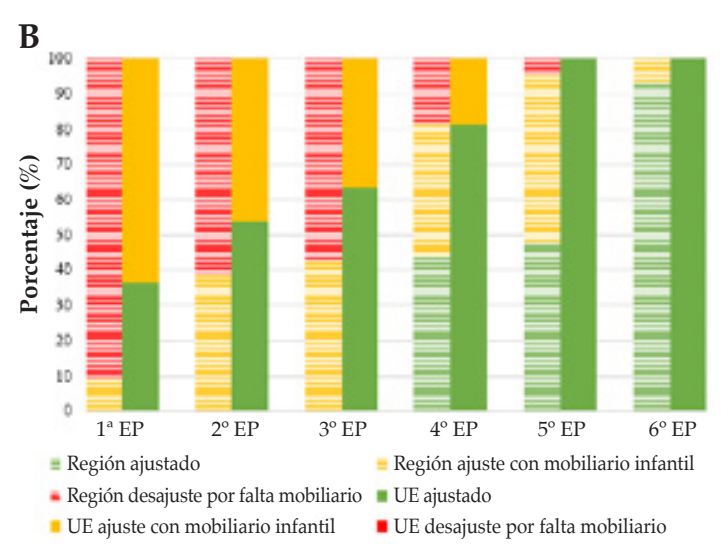


seguir las tallas del catálogo de la UE, ${ }^{12}$ el cual permite adecuar tanto las sillas como las mesas a las características de todo el alumnado. Así, serían necesarias cinco tallas para sillas $(26,31$, $35,38$ y 43$)$ y cuatro tallas para las mesas (46, 53,59 y 64). Con el catálogo actual de la región de Galicia, ${ }^{13}$ solo es posible el ajuste total en $5^{\circ}$ y $6^{\circ}$ de la EP (10 a 12 años). En el resto de los cursos, se tendría que recurrir a mobiliario no destinado a la EP. Como caso más destacable, señalar que la totalidad del alumnado de $1^{\circ}$ de la EP utiliza mobiliario inadecuado. Si a $1^{\circ}$ EP se le asignara el mobiliario destinado por normativa a educación infantil, se podría ajustar tan solo al $45,5 \%$ de los estudiantes en la silla y al 9,1\% en la mesa. El alumnado restante no encontraría en las tallas de la normativa de mobiliario de Galicia una silla y mesa acorde a sus características antropométricas. Si el centro dispusiera de muebles según la normativa de la UE, sí podría hacer una distribución apropiada a la totalidad de sus estudiantes.

A su vez, se observaron diferencias estadísticamente significativas respecto a las tallas de silla y mesa que debe emplear el alumnado por curso, por lo que no se puede determinar una única talla por curso o edad, sino más bien establecer una propuesta de tamaño de mobiliario por nivel madurativo. Estos hallazgos coinciden con las conclusiones de otras investigaciones ${ }^{22,25}$ y evidencian la variabilidad de dimensiones antropométricas del alumnado de la EP, siendo necesaria tanto en silla como en mesa, entre dos $\mathrm{y}$ tres tallas diferentes en cada curso, o mobiliario regulable..$^{24,26}$

\section{Limitaciones}

Existen ciertas debilidades metodológicas, como el reducido tamaño muestral, perteneciente a un único colegio público, o los análisis comparativos entre los diferentes grupos (al ser pequeños) tanto al analizar los diferentes cursos como el sexo de los participantes. Por tanto, deben ser tenidas en cuenta, pues limitan la interpretación y transferencia de los resultados presentados, al no ser una muestra representativa de toda la región. Tampoco se utilizó ningún instrumento que permitiera detectar dolencias músculo-esqueléticas. Sin embargo, los hallazgos podrían ser un indicador de un problema similar en este y otros países, por lo que resulta necesario realizar estudios con un número más grande de estudiantes.

\section{CONCLUSIONES}

Existe un desajuste entre las características antropométricas del alumnado y las medidas del mobiliario escolar, con un elevado porcentaje de estudiantes que se sientan en una silla y emplean una mesa, demasiado grandes.

El catálogo de tallas regional que se está utilizando en la actualidad es inadecuado, al no seguir el criterio de ajuste antropométrico. En cambio, las tallas indicadas en el catálogo europeo se adaptan correctamente a la muestra de referencia, por lo que es necesario utilizar varias tallas por curso o mobiliario regulable para adaptarse a las características antropométricas de todo el alumnado.

\section{REFERENCIAS}

1. Cooper AR, Goodman A, Page AS, Sherar LB, et al. Objectively measured physical activity and sedentary time in youth: The International children's accelerometry database (ICAD). Int J Behav Nutr Phys Act. 2015; 12:113.

2. Janssen X, Mann KD, Basterfield L, Parkinson KN, et al. Development of sedentary behavior across childhood and adolescence: Longitudinal analysis of the Gateshead Millennium Study. Int J Behav Nutr Phys Act. 2016; 13:88.

3. Mooses K, Mägi K, Riso EM, Kalma M, et al. Objectively measured sedentary behaviour and moderate and vigorous physical activity in different school subjects: a crosssectional study. BMC Public Health. 2017; 17(1):108.

4. Ridgers ND, Salmon J, Ridley K, O'Connell E, etal. Agreement between activPAL and ActiGraph for assessing children's sedentary time. Int J Behav Nutr Phys Act. 2012; 9:15.

5. Castellucci HI, Arezes PM, Molenbroek JFM, de Bruin R, Viviani C. The influence of school furniture on students' performance and physical responses: results of a systematic review. Ergonomics. 2016; 60(1):93-110.

6. Mura G, Vellante M, Nardi A, Machado S, Carta M. Effects of school-based physical activity interventions on cognition and academic achievement: a systematic review. CNSNeurol Disord Drug Targets. 2015; 14(9):1194-208.

7. Fettweis T, Demoulin C, Vanderthommen M. Influence de la position assise d'enfants sur leurs capacités cognitives. Kinésithérapie. 2013; 13(136):20-5.

8. Wick K, Faude O, Manes S, Zahner L, Donath L. I can stand learning: A controlled pilot intervention study on the effects of increased standing time on cognitive function in primary school children. Int J Environ Res Public Health. 2018; 15(2):356.

9. Instituto Nacional de Normalización. Norma chilena 2566. Mobiliario escolar de silla y mesas escolares y requisitos dimensionales. Santiago de Chile: INN; 2002.

10. JapaneseStandards Association. JISS1021 School furnitureDesk and chairs for general learning space. Tokyo: Japanese Industrial Standards; 2011.

11. Korean Industrial Standards. KS G 2010:2015 Chairs and Tables for Educational Institutions. Korea: KIS; 2015.

12. European Committee for Standadization CEN. Furniture - Chairs and tables for educational institutions - Part 1: Functional dimensions. Brussels: CEN; 2015.

13. Xunta de Galicia. Equipamento de centros: catálogos xerais [Internet]. Galicia: Conselleria de Educación, Universidade e Formación Profesional; 2017 [Acceso: 1 de marzo de 2021]. Disponibleen:https: / / www.edu.xunta.gal/portal/ node/495 
14. Harriss DJ, Atkinson G. Ethical Standards in Sport and Exercise Science Research: 2016 Update. Int J Sports Med. 2015; 36(14):1121-4.

15. Bragança S, Arezes P, Carvalho M, Ashdown SP, et al. A comparison of manual anthropometric measurements with Kinect-based scanned measurements in terms of precision and reliability. Work. 2018; 59(3):325-39.

16. ISO. ISO 7250-1: Basic human body measurements for technological design-Part 1: Body measurement definitions and landmarks. $2^{\text {nd }}$ ed. Geneva: International Organization for Standardization; 2017.

17. Castellucci HI, Arezes PM, Molenbroek JFM. Applying different equations to evaluate the level of mismatch between students and school furniture. Appl Ergon. 2014; 45(4):1123-32.

18. Brewer JM, Davis KG, Dunning KK, Succop PA. Does ergonomic mismatch at school impact pain in school children? Work. 2009; 34(4):455-64.

19. Parvez MS, Parvin F, Shahriar MM, Kibria G. Design of Ergonomically Fit Classroom Furniture for Primary Schools of Bangladesh. J Eng (United Kingdom). 2018; 2018:3543610.

20. Castellucci HI, Catalán M, Arezes PM, Molenbroek JFM. Evaluation of the match between anthropometric measures and school furniture dimensions in Chile. Work. 2016; 53(3):585-95.
21. Manrique-Olivares D, Quispe-Montoya K. ¿Es el mobiliario escolar adecuado para los estudiantes? Evaluación de una muestra de escolares peruanos. Arch Argent Pediatr. 2016; 114(1):e70.

22. Macedo AC, Morais A V, Martins HF, Martins JC, et al. Match between classroom dimensions and students' anthropometry: Re-equipment according to european educational furniture standard. Hum Factors. 2015; 57(1):4860.

23. Da Silva LB, Coutinho AS, Da Costa Eulálio EJ, Soares EVG. School furniture and work surface lighting impacts on the body posture of Paraíba's public school students. Work. 2012; 42(4):579-87.

24. Cantin N, Delisle I, Baillargeon M. Reducing ChildFurniture Incompatibility in Primary Schools. J Occup Ther Sch Early Interv. 2019; 12(2):200-9.

25. Lee Y, Yun MH. Evaluation of the guidelines and children's ability to select the anthropometrically recommendable height of school furniture: A case study of Korean primary school children. Work. 2019; 64(3):427-38.

26. Yanto, Lu CW, Lu JM. Evaluation of the Indonesian National Standard for elementary school furniture based on children's anthropometry. Appl Ergon. 2017; 62:168-81 